\title{
Patient Preferences in Selecting a Dentist: Survey Results from the Urban Population of Riyadh, Saudi Arabia
}

\author{
${ }^{1}$ Sahar Bin Huraib, ${ }^{2}$ Nadia Al Nahas, ${ }^{3}$ Hana O Al-Balbeesi, ${ }^{4}$ Faida Moawia Abu-Aljadayl \\ ${ }^{5}$ Sajith Vellappally, ${ }^{6}$ Anil Sukumaran
}

\begin{abstract}
Introduction: Awareness of gender- or nationality-driven preconceptions can help dentists to have a better interpretation of the dentist-patient relationship. It is even more noteworthy to understand these predilections in Saudi society, where women and men are usually segregated due to religion- and culturebased considerations. This study is one of the first to explore the preferences of patients when selecting a dentist with respect to gender and nationality in the city of Riyadh, Saudi Arabia.
\end{abstract}

Materials and methods: A total of 445 community residents residing in Riyadh were randomly selected for a cross-sectional study. The participants completed a survey designed to assess which of two factors (gender and/or nationality) were perceived as most relevant in choosing a dentist. Statistical analysis of the data was performed using the SPSS 11.5 software.

Results: Female participants did not show any preference for the gender of the dentist, whereas $40 \%$ of the male participants preferred a male dentist. Participants also favored male dentists in the fields of oral surgery $(78.9 \%)$, implants $(74.1 \%)$, endodontics (67.5\%), orthodontics (65.8\%) and prosthodontics (64.2\%). An exception was noted in pediatric dentistry, for which female dentists were favored by $52.8 \%$ of the participants. Additionally, most (66.1\%) participants did not have any preference for the nationality of the dentist.

Conclusion: Riyadh residents showed a general preference for a male dentist but demonstrated no preference for nationality when selecting a dentist.

${ }^{1}$ Department of Dental Health, Dental Public Health, College of Applied Medical Sciences, King Saud University, Riyadh Saudi Arabia

${ }^{2}$ Department of Periodontics and Community Dentistry, College of Dentistry, King Saud University, Riyadh, Saudi Arabia

${ }^{3}$ Department of Pediatric Dentistry and Orthodontics, College of Dentistry, King Saud University, Riyadh, Saudi Arabia

${ }^{4}$ Department of Infection Control, Alyamamah Hospital, Ministry of Health, Riyadh, Saudi Arabia

${ }^{5}$ Department of Dental Health, Dental Biomaterials Research Chair, College of Applied Medical Sciences, King Saud University, Riyadh, Saudi Arabia

${ }^{6}$ Department of Periodontics and Community Dentistry, College of Dentistry, King Saud University, Riyadh, Saudi Arabia

Corresponding Author: Sahar Bin Huraib, Consultant Department of Dental Health, Dental Public Health College of Applied Medical Sciences, King Saud University PO Box 92093, Riyadh 11673, Saudi Arabia, e-mail: sbinhuraib1@kus.edu.sa
Keywords: Dentist-patient relationship, Dentist, Gender preference, Saudi Arabia, Patient satisfaction, Specialties.

How to cite this article: Huraib SB, AI Nahas N, Al-Balbeesi HO, Abu-AljadaylFM, VellappallyS, Sukumaran A.PatientPreferences in Selecting a Dentist: Survey Results from the Urban Population of Riyadh, Saudi Arabia. J Contemp Dent Pract 2015;16(3): 201-204.

\section{Source of support: Nil}

Conflict of interest: None

\section{INTRODUCTION}

Demographic factors, such as gender, age, and nationality can contribute to patient preferences for dental and other healthcare providers. Patient preferences and provider characteristics may have implications for the treatment received. Choosing a dentist can be difficult. People's personalities, including their ways of thinking and perspectives differ and might influence the decisionmaking process. Several factors have been considered when patients choose a dentist or medical practitioner. ${ }^{1,2}$ Studies have suggested that patients prefer practitioners from their own ethnic background. ${ }^{3}$ This concordance is rooted in the perception of having a better communication, which promotes improved patient participation, understanding and tolerance in the consultation. ${ }^{4}$ However, other researchers have found that more patients were accepting practitioners from other ethnic backgrounds, particularly if the practitioner had a positive and welcoming personality. ${ }^{5}$

For several years, the demographic profile of healthcare practitioners in Saudi Arabia has become increasingly diverse. ${ }^{6}$ The same diversity in gender and nationality has been observed among dental practitioners in Saudi Arabia. Recent estimates from the Ministry of Health indicate a ratio of 1:2 for male to female dentists and a ratio of 1:2 for Saudi to non-Saudi dentists. This diversity brings important challenges in the delivery of effective, culturally sensitive, and respectful services to patients seeking treatment. ${ }^{6}$ This issue becomes more important in Saudi society, where women and men are usually segregated due to religious and cultural considerations.? In the medical field, studies have shown that female practitioners communicate more with their patients during 
the collection of information, sharing and discussion of psychosocial topics, partnership building, and encouragement of preventive health practices. ${ }^{8}$ This style reflects a closer aligned therapeutic environment with greater patient engagement and partnership, whereas male practitioners tend to devote more time to technical practice behaviors. $^{9}$

Academic and government institutions bear the responsibility of meeting the healthcare needs of the public by providing a workforce that is sufficient in both number and quality. ${ }^{10}$ Unfortunately, very few studies have examined the effects that practitioner demographics have on patient preferences. ${ }^{6}$ Research findings have revealed that knowing how patients make certain healthcare decisions can allow for a better interpretation of the dentist-patient relationship. ${ }^{11}$ Awareness of gender- and ethnic-driven preconceptions can help policy makers improve the services available to patients in both the public and private sectors. ${ }^{12}$

Social and cultural factors might influence the selection of a medical practitioner in Saudi Arabia. The smaller number of foreign nationals in various fields of medical practice in Saudi Arabia could make the decision-making process more complex. There is a scarcity in the literature on patient preferences in choosing a dentist in Saudi Arabia. Hence, the present study aims to evaluate patient preferences in selecting a dentist in Riyadh, an urban city in Saudi Arabia.

\section{MATERIALS AND METHODS}

Ethical approval for the study was obtained from the College of Dentistry Research Centre (CDRC), King Saud University. Participants were invited to take part in this study and were not remunerated for participation. Verbal consent was secured from participants. A total of 445 residents from Riyadh were selected for this cross-sectional study. A pilot study was conducted to identify any shortcomings. Participants provided their demographic information, including gender, age, ethnicity and marital status, which were considerably diverse. Statistical analysis of the data was performed using the SPSS 11.5 software, and univariate analysis

Table 1: Demographic characteristics of the study population

\begin{tabular}{llll}
\hline & & Number & Percentage (\%) \\
\hline Gender & Male & 136 & 31 \\
\multirow{3}{*}{ Nationality } & Female & 309 & 69 \\
& Saudi & 427 & 96 \\
\multirow{3}{*}{ Education } & Non-Saudi & 18 & 4 \\
& Graduates & 219 & 49 \\
& Non & 226 & 51 \\
\hline
\end{tabular}

was used to study the independent effect of the different variables.

\section{RESULTS}

Sample characteristics: The age distribution of the participants ranged from 15 to over 70 years with the largest age group (26.1\%) being 25 to 30 years. Among the participants, 69\% (309) were female, 96\% were Saudi, and $49 \%$ held a university degree (Table 1 ).

Distribution of patient preferences: The results indicate a statistically significant difference $\left(\chi^{2}=15.81\right.$ and $\left.p<0.05\right)$ between the responses of males and females in terms of selecting the gender of the dentist. Though the females did not show any preference for the sex of the dentist, $40 \%$ of the male participants preferred a male dentist (Graph 1). A similar statistically significant response $\left(\chi^{2}=40.196\right.$ and $p<0.05$ ) was observed when $16.9 \%$ of females and $2.3 \%$ of males preferred a male dentist for treating their family members. When further probed about gender preferences within the different specialties of dentistry, most study participants favored a male dentist in the fields of oral surgery $(78.9 \%)$, implants $(74.1 \%)$, endodontics $(67.5 \%)$, orthodontics (65.8\%) and prosthodontics (64.2\%). An exception was noted in pediatric dentistry, for which female dentists were favored by $52.8 \%$ of the participants (Table 2 and Graph 2).

Of the 445 participants, $19 \%$ preferred to be treated by a Saudi dentist and $14 \%$ by a non-Saudi dentist. The remaining 294 (67\%) participants did not show any preference in terms of nationality of the dentist (Graph 3). Additionally, the majority (61.9\%) of participants from both genders preferred the private sector's dental services rather than the government dental services.

\section{DISCUSSION}

Most studies by social scientists related to gender or nationality stereotypes have had direct implications for clinical settings. ${ }^{13}$ However, few studies have investigated whether these findings mirror the traditional perceptions in dentistry. ${ }^{14}$ Awareness of gender- or nationality-driven

Table 2: Patient preference for dentist's gender based on dental specialties

\begin{tabular}{lll}
\hline Specialty & Male (\%) & Female (\%) \\
\hline Endodontics & $114(67.5)$ & $55(32.5)$ \\
Oral surgery & $127(78.9)$ & $34(21.1)$ \\
Orthodontics & $104(65.8)$ & $54(34.2)$ \\
Prosthodontics & $102(64.2)$ & $57(35.8)$ \\
Periodontics & $117(74.1)$ & $41(25.9)$ \\
Implant dentistry & $80(51.6)$ & $75(48.4)$ \\
Restorative dentistry & $87(54.4)$ & $73(47.6)$ \\
Pediatric dentistry & $77(54.4)$ & $87(52.8)$ \\
\hline
\end{tabular}




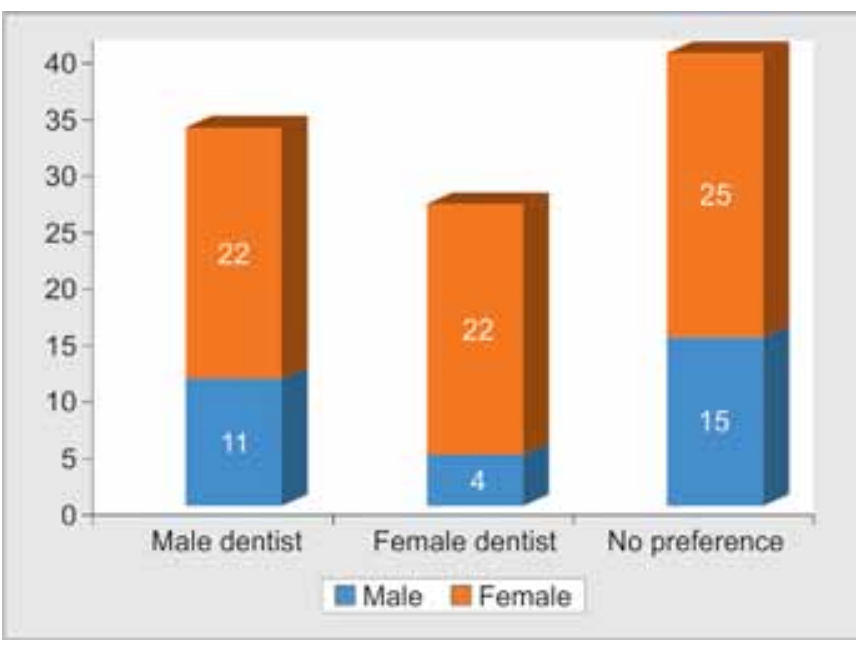

Graph 1: Patient preference for the gender of the dentist

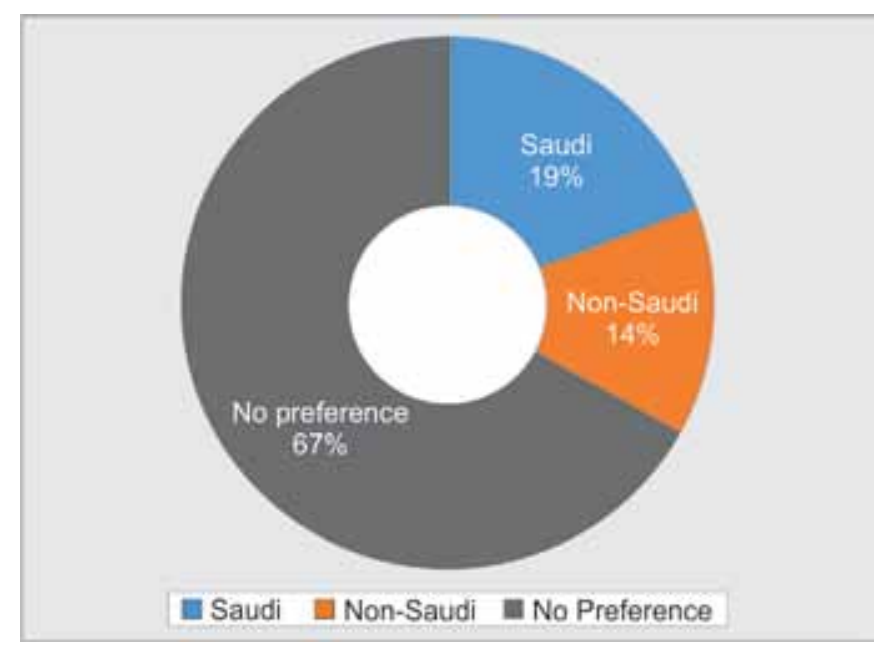

Graph 3: Patient preference for the nationality of the dentist

preconceptions can help dentists anticipate patient expectations, which can play a role in the clinician-patient relationship. ${ }^{9}$ It is even more noteworthy to understand these predilections in Saudi society, where women and men are usually segregated due to religious and cultural considerations. ${ }^{15}$ Additionally, patient preferences in selecting a dentist according to nationality can provide important insights into the psychosocial parameters involved in dental care, particularly in Saudi Arabia, where a large number of practicing dentists are foreign nationals. ${ }^{16}$ This study is one of the first in Saudi Arabia to explore the preference of patients in the selection of a dentist with respect to gender and nationality.

In the present study, the majority of females did not show any gender preference, whereas the participating males generally preferred a male dentist, a finding that is in agreement with most previous studies. ${ }^{17}$ Nevertheless, a significant number of participants favored male dentists for treating their family members. Furthermore, with respect to different specialties within dentistry, participants expressed a preference for male dentists regard-

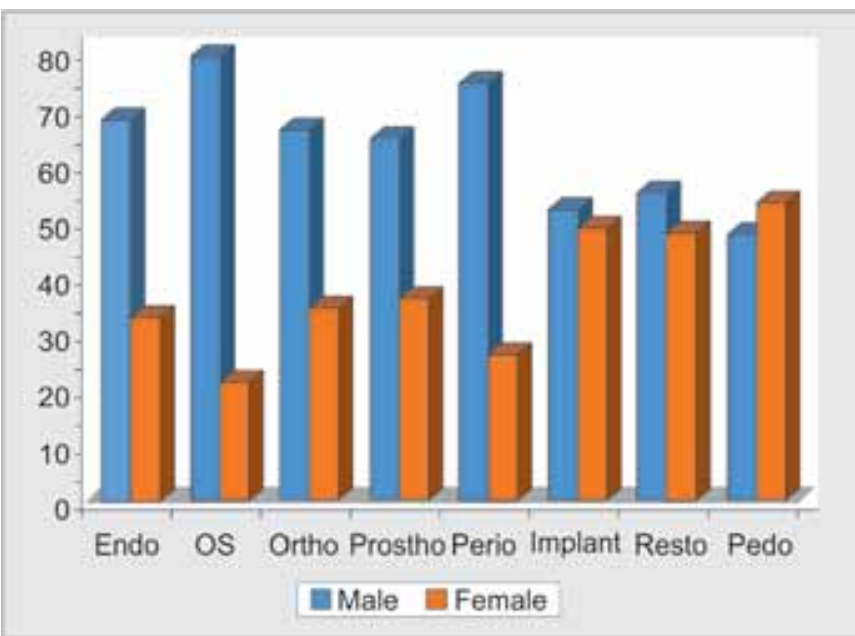

Graph 2: Patient preference for dentist's gender based on dental specialties

less of their own gender, except for pediatric dentists, a finding that is also consistent with previous studies. ${ }^{13,18,19}$ This could be attributed to the female practitioners having greater empathy traits, such as more humanness, compassion, communication through nonverbal cues, caring, patience and an expressive attitude towards patients. ${ }^{1720}$ The preference for a male dentist might be due to various factors, such as the sense of more competency in male compared with female practitioners or the general notion of male dentists as more career-oriented and professional than their female counterparts. ${ }^{12}$ Most participants reasoned from their previous experiences and dentist's expertise to explain their bias towards male dentists, thus validating the above assumptions. However, it is interesting to note that more than a quarter of the sample participants justified their selection using religious and cultural values.

The results of this study indicate that most of the sample participants were not concerned about the nationality of the dentist. This finding is in accordance with earlier studies. ${ }^{21,22}$ Participants did not rate the nationality-concordant provider higher on any measure, thus emphasizing the importance of expertise. Another key point was that all participants had a high educational background, with the majority having at least a bachelor's degree, which could help explain their preferences.

The communication between medical practitioner and patient remains the core factor in building confidence in patients. ${ }^{23}$ Studies have shown that the physician-patient communication has a significant influence on the outcome of patient care, including patient satisfaction, compliance with treatment, recall and understanding of medical information, and coping with disease. ${ }^{24,25}$ Communication could be a key factor for choosing a Saudi as a dentist.

Several factors limit the generalization of this research. Because the study is based on a small sample, 
we cannot generalize the findings to a larger population. In addition, the majority (70\%) of respondents were female. Furthermore, the items included in the survey were by no means comprehensive. Other questions might have revealed whether gender-based assessments of dentists could be influenced by a myriad of other factors, such as the office environment or the interaction between the clinician's gender and communication style. ${ }^{26,27}$

\section{CONCLUSION}

The study reveals a general bias towards male dentists irrespective of the patient's gender but no preference over the nationality of the dentist. These findings contradict the gender-nationality concordance hypothesis. Previous experience and religious and cultural values have been suggested as the reasons for selecting a male dentist. However, further research on other factors should be conducted to allow for a broader understanding of patient preferences as they play a major role in the dentist-patient relationship.

\section{REFERENCES}

1. Cleary PD, McNeil BJ. Patient satisfaction as an indicator of quality care. Inquiry 1988. p. 25-36.

2. Weiss GL. Patient satisfaction with primary medical care. Evaluation of sociodemographic and predispositional factors. Med Care 1988;26(4):383-392.

3. Hardie R, Ransford E, Zernik J. Dental patients' perceptions in a multiethnic environment. J Calif Dent Assoc 1995;23(12): 77-80.

4. van Ryn M, Burke J. The effect of patient race and socioeconomic status on physicians' perceptions of patients. Soc Sci Med 2000;50(6):813-828.

5. Gerbert B, Berg-Smith S, Mancuso M, Caspers N, Danley D, Herzig K, et al. Video study of physician selection: preferences in the face of diversity. J Fam Pract 2003;52(7):552-559.

6. Swami V, McClelland A, Bedi R, Furnham A. The influence of practitioner nationality, experience, and sex in shaping patient preferences for dentists. Int Dent J 2011;61(4):193-198.

7. Baki R. Gender-segregated education in Saudi Arabia: its impact on social norms and the Saudi labor market. Education Policy Analysis Archives 2004;12(28):n28.

8. Street RL. Communication in medical encounters: an ecological perspective. Handbook of Health Communication 2003. p. 63-89.

9. Bertakis KD, Azari R. Patient-centered care: the influence of patient and resident physician gender and gender concordance in primary care. J Womens Health (Larchmt) 2012;21(3): 326-333.
10. Khan T, Thomas LS, Naidoo S. Analysing post-apartheid gender and racial transformation in medical education in a South African province. Glob Health Action 2013;6:19810.

11. Cooper-Patrick L, Gallo JJ, Gonzales JJ, Vu HT, Powe NR, Nelson C, et al. Race, gender, and partnership in the patientphysician relationship. JAMA 1999;282(6):583-589.

12. Smith MK, Dundes L. The implications of gender stereotypes for the dentist-patient relationship. J Dent Educ 2008; 72(5):562-570.

13. Newton JT, Davenport-Jones L, Idle M, Patel M, Setchell A, Turpin C. Patients' perceptions of general dental practitioners: the influence of ethnicity and sex of dentist. Social Behavior and Personality Int J 2001;29(6):601-606.

14. Maccoby EE. Gender and relationships: a developmental account. Am Psychol 1990;45(4):513-520.

15. Al Faris E, Al Hamdan N, Babelli DM, Al Nour MAB, Al Dahry S, Al Zaidan H, et al. The impact of the doctor's gender on the doctor-patient relationship in a Saudi obstetric and gynaecology clinic. Saudi Med J 1994;15(6):450-455.

16. Ahmad WI, Kernohan EE, Baker MR. Patients' choice of general practitioner: importance of patients' and doctors' sex and ethnicity. Br J Gen Pract 1991;41(349):330-331.

17. Fennema K, Meyer DL, Owen N. Sex of physician: patients' preferences and stereotypes. J Fam Pract 1990;30(4):441-446.

18. Patir Munevveroglu A, Balli Akgol B, Erol T. Assessment of the feelings and attitudes of children towards their dentist and their association with oral health. ISRN Dent 2014;2014: 867234.

19. Bare LC, Dundes L. Strategies for combating dental anxiety. J Dent Educ 2004;68(11):1172-1177.

20. Sinkford JC, Valachovic RW, Harrison S. Advancement of women in dental education: trends and strategies. J Dent Educ 2003;67(1):79-83.

21. Bertakis KD. Does race have an influence on patients' feelings toward physicians? J Fam Pract 1981;13(3):383-387.

22. Aruguete MS, Roberts CA. Participants' ratings of male physicians who vary in race and communication style. Psychol Rep 2002;91(3 Pt 1):793-806.

23. Shaw A, Ibrahim S, Reid F, Ussher M, Rowlands G. Patients' perspectives of the doctor-patient relationship and information giving across a range of literacy levels. Patient Educ Couns 2009;75(1):114-120.

24. Main CJ, Buchbinder R, Porcheret M, Foster N. Addressing patient beliefs and expectations in the consultation. Best Pract Res Clin Rheumatol 2010;24(2):219-225.

25. Hagihara A, Tarumi K. Doctor and patient perceptions of the level of doctor explanation and quality of patient-doctor communication. Scand J Caring Sci 2006;20(2):143-150.

26. Bender DJ. Patient preference for a racially or genderconcordant student dentist. J Dent Educ 2007;71(6):726-745.

27. Schmid Mast M, Hall JA, Roter DL. Disentangling physician sex and physician communication style: their effects on patient satisfaction in a virtual medical visit. Patient Educ Couns 2007;68(1):16-22. 